\title{
Changing of the guard at The Journal of Headache and Pain
}

As our readers can see, The Journal of Headache and Pain is experimenting a phase of evolution, from grub to chrysalis. Even if the neonatal phase has only just passed, it is still a reminder of the actual adolescent phase which presented itself full of fears, courage, uncertainties and dreams. It is right that it should be so. The will to grow is always full of such emotions.

The necessity to bid an almost simultaneous and sad farewell to Virgilio Gallai (as Founding Editor-inChief) and to Francomichele Puca (as Founding Associate Editor) has left a feeling of deep loss among our ranks. On the humus (breeding ground) of different views and on a normal and healthy exchange of ideas has bred a solid reality which has been nurtured and taken root. I remember how our editorial initiative was considered a too ambitious project, even opposed, and can only hope that it was just a natural response to the res nova. Perseverance towards an ideal has won over scepticism. Our idea was to propose to the international scientific world a side window to the areas of headache and pain and it continues to gradually develop without interference with old and solid editorial realities. If we all support the idea that today only onequarter of patients suffering from migraine receive adequate diagnosis and cure, we cannot deny that there exists space for stimulating products to be investigated or that clinical, social or basic research in this area of medicine has not been saturated. No, indeed!

Mention must also be made of the Italian Society for the Study of Headaches, the raison d'etre of this Journal. This scientific society is now well-established, and has reached its twenty-eighth year since foundation without fears of international integration, and is also the only nonAnglophone scientific society to have twice hosted the congress of the International Headache Society, in 1987 in Florence and in 2003 in Rome.

Now, The Journal of Headache and Pain is to embody a strategic priority for the next five years. It is not my desire to appear before you today as a neurotic innovator - a too-often practice for incoming Editors-in-Chief outlining a list of what should be modified, changed, innovated. The natural, human desire to leave forever a personal imprint often brings chaos to valuable works that maybe only needed a little restoration.

Instead I would like to reiterate to all of you what I stated in the strategic proposition which accompanied my application for this position. I think that the future of this Journal must base itself on four solid cornerstones which have already been in operation for some time [1-3]: the internal joint leadership of the Editorial Board concerned with the management of sub- 
mitted manuscripts; the exclusive management via the Web of these manuscripts; the enhancement of interest towards the neglected topic of the impact of headache disorders on public health; and promotion of the multidisciplinary study of painful pathologies.

On the other hand, the recent International Classification of Functioning, Disability and Health (ICF) of the World Heath Organization (WHO) has stressed a priority that no one can ignore [4]. We cannot voice sufficiently loudly our sense of achievement that the area of headache is no longer the "Cinderella" within the panorama of clinical medicine, considering that the ICF has placed migraine in the nineteenth position among the most debilitating illnesses. Instead, we must busy ourselves in order to favor the growth of the culture of the single and the collective in the area of social medicine applied to headache and painful syndromes, in their formulation and research [5].

Regarding the new plan for the Journal, I would like to offer in each issue a concise opening stage to the sector leaders who will be invited to express their opinions regarding evaluation, critique, indication of tendencies, innovations. The policy regarding the sections dedicated to specific subjects will continue as in the past. In the wake of the pioneer section "On the Web" I think that it is now time for a "Public Health Section" and an interesting and culturally lively "Historical Section". The medicine of headache is a many-faceted area and as such I feel it must be realized in all its various connotations. These new sections will be entrusted to recognized experts in their respective fields.

Above all, I hope that in my role as Editor-in-Chief I may serve as a coordinating link of scientific excellence and so enable the Senior Editor-inChief, the Deputy Editor and Associate Editors to work with maximum directional autonomy within the Journal. Finally, the sincere wish that I offer to the entire Editorial Board and to all readers is that The Journal of Headache and Pain may represent not only a simple gym where one can practice when one feels like it, but instead a green rugby field where we may confront each other, even roughly, but with well known rules respected by all.

The continuous tracking of the status of one's own manuscript, the possibility to choose among one of three referees, and the maximum sense of timing of the publication represent some of the new keys in the editorial offer we are making to the scientific world. We will be able to give a stage to the development of the tripod of the science of headache: scientific research, academic teaching methods and the formation of new researchers. Thus we will be able to contribute in a nonunrealistic extent and thereby enable us to render the area of headache medicine the dignity of a new discipline.

I hope that after having inherited a chrysalis, in my turn I may be able to leave to my future successor a splendid multicolored butterfly free to fly.

\section{Paolo Martelletti Editor-in-Chief}

\section{References}

1. Martelletti P (2003) Web-based submission of manuscripts to The Journal of Headache and Pain. J Headache Pain 4:42

2. Martelletti P, Leonardi M (2003) The global impact of migraine. J Headache Pain 4:S1-S2
3. Martelletti P (2002) The unfinished war. J Headache Pain 3:115-116

4. - (2001) International classification of functioning, disability and health. World Health Organization, Geneva (available at http://www3. who.int/icf/icftemplate.cf $\mathrm{m}$, viewed 9 March 2004)
5. - (2000) Headache disorders and public health. Education and management implication. World Health Organization, Geneva (WHO.MSD.MBD/00.9) 